\title{
一锅法合成含两种桥联链的杯[4]双冠醚和双杯[4]冠醚
}

\author{
叶进启 ${ }^{a} \quad$ 杨发福 ${ }^{*, a}, b \quad$ 张篠逸 $^{a} \quad$ 焦自玉 ${ }^{a} \quad$ 郭红玉 $^{a}$ \\ ( ${ }^{a}$ 福建师范大学化学与材料学院 福州 350007) \\ ( ${ }^{b}$ 福建师范大学福建省高分子材料重点实验室 福州 350007)
}

\begin{abstract}
摘要 报道了 “一锅法” 合成含两种桥联链的新型杯 [4]双冠醚和双杯 [4]冠醚. 杯[4]芳烃先与 $N, N^{\prime}$-乙撑基-二(2-氯乙酰 胺)发生 “ $1+1$ ” 缩合反应，然后直接加入三甘醇双对甲苯磺酸酯继续进行 “ $2+2$ ” 缩合反应，合成了含两种桥联链的 新型双杯 [4]冠醚 4. 按照相似程序, 杯[4]芳烃先后与 $N, N^{\prime}$-乙撑基-二(2-氯乙酰胺)、澳乙酸乙酯和二乙烯三胺反应, 得 到含两种桥联链的新型杯 [4]双冠醚 5. 化合物 5 进一步与异硫氧酸苯酯反应合成带硫䐖支链的杯 [4]双冠醚 6. 所有新化 合物的结构与构象经元素分析、质谱、核磁共振谱等表征证实.
\end{abstract}

关键词 杯[4]冠醚; 双杯[4]芳烃; 合成; 一锅法

\section{One-Pot Syntheses of Calix[4]-bis-crowns and Biscalix[4]crown with Two Kinds of Bridging Chains}

\author{
Ye, Jinqi ${ }^{a} \quad$ Yang, Fafu $^{*, a, b} \quad$ Zhang, Xiaoyi $^{a} \quad$ Jiao, Ziyu $^{a} \quad$ Guo, Hongyu ${ }^{a}$ \\ ( ${ }^{a}$ College of Chemistry and Materials, Fujian Normal University, Fuzhou 350007) \\ ( ${ }^{b}$ Fujian Key Laboratory of Polymer Materials, Fujian Normal University, Fuzhou 350007)
}

\begin{abstract}
The one-pot synthesis of novel calix[4]-bis-crowns and biscalix[4]crown with two kinds of bridging chains was described. Calix[4] arene was firstly reacted with $N, N$ '-ethylenebis(2-chloroacetamide) in " $1+1$ " condensation, and then triethylene glycol ditosylate was added directly to give a new biscalix[4]crown 4 with two kinds of bridging chains in " $2+2$ " condensation. According to similar procedure, by successively reacting calix[4]arene with $N, N^{\prime}$-ethylenebis(2-chloroacetamide), ethyl bromoacetate and diethylenetriamine, a new calix[4]-bis-crowns 5 with two kinds of bridging chains was prepared. Further reacting compound 5 with phenyl isothiocyanate afforded calix[4]-bis-crowns 6 with thiourea branched chain. The structures and conformation of new compounds were characterized by elemental analyses, ESI-MS, ${ }^{1} \mathrm{H}$ NMR, etc.
\end{abstract}

Keywords calix[4]crown; biscalix[4]arene; synthesis; one-pot

杯芳烃是超分子化学中继冠醚和环糊精之后广受 瞩目的第三代主体分子，其作为有效的合成平台，通过 适当的化学修饰可以构筑对特定客体具有识别能力的 新型主体分子 ${ }^{[1 \sim 3]}$. 杯芳烃冠醚是杯芳烃衍生物中重要 的一类，由于杯芳烃单元与冠醚单元能构筑新的三维包 合空腔并存在协同识别作用，常展现出特异的客体识别 能力. 目前, 一系列杯芳烃冠醚和杯芳烃氮、硫杂冠醚都 已被报道 ${ }^{[4-9]}$. 杯芳烃双冠醚和双杯芳烃冠醚是杯芳烃冠 醚中结构较为复杂的一类, 由于多桥联链与杯芳烃骨架
围成多配合空腔，可以同时识别多个客体分子，颇受研 究者关注 ${ }^{[10]}$. 综合文献来看, 杯 [4]双冠醚和双杯 [4]冠醚 的合成方法主要有两种: 一是一步法: 杯芳烃与多倍的 双官能团桥联试剂直接反应，得到的是含同种桥联链的 杯[4]双冠醚或双杯 [4]冠醚，这一方法步骤虽简单，但 副产物多, 分离困难, 产率较低 ${ }^{[11 ~ 14]}$; 二是多步法: 通 过分步骤先后与相同或不同的双官能团试剂反应合成 含相同或不同桥联链的杯 [4]双冠醚或双杯 [4]冠醚，这 种方法步骤繁琐，总产率不高 ${ }^{[15 ~ 18]}$. 本文中，我们利用

\footnotetext{
*E-mail: yangfafu@fjnu.edu.cn

Received May 29, 2011; revised August 18, 2011; accepted September 5, 2011.

Project supported by the National Natural Science Foundation of China (No. 20402002), the Natural Science Foundation of Fujian Province (No. 2011J01031), the Program for Excellent Young Researchers in University of Fujian Province (No. JA10056), and the Project of Fujian Provincial Department of Education (No. JA11044).

国家自然科学基金(No. 20402002)、福建省自然科学基金(No. 2011J01031)、福建省高校杰出青年科研人才培育计划(No. JA10056)、福建省教育厅重
} 点(No. JA11044)资助项目. 
“一锅法”，集合一步法和分步法的各自优势，采取不分 离中间产物、先后加入不同桥联试剂的方法, 制备了三 例含不同桥联链的新型杯 [4]双冠醚和双杯 [4]冠醚, 产 率中等, 为合成这类结构复杂的主体分子提供了新的思 路. 合成路线如 Scheme 1 所示.

\section{1 实验部分}

\section{1 仪器与试剂}

Varian Unity $500(500 \mathrm{MHz})$ 核磁共振仪; DECAX-30000 LCQ Deca XP ESI-MS 质谱仪(甲醇作溶剂); Reichert 7905 熔点仪(温度未校正); Vario EL III 自动元 素分析仪. 碳酸钾、丙酮、二氧六环均经标准程序干燥 后使用, 其它试剂为分析纯直接使用. 对叔丁基杯[4]芳 烃 1 和母体杯芳烃 2 按文献方法合成 ${ }^{[1,2]}$.

\section{2 合成}

\subsection{1 杯[4]氮杂冠醚 3 的合成}

参考文献[5]方法, 氮气保护下，向 $100 \mathrm{~mL}$ 三口烧 瓶中加入 $0.424 \mathrm{~g}(1 \mathrm{mmol})$ 去叔丁基杯[4]芳烃 $2,0.23 \mathrm{~g}$ (1.1 mmol) $N, N^{\prime}$-亚乙基-二(2-氯乙酰胺), $30 \mathrm{~mL}$ 干燥丙

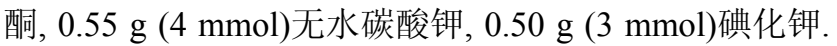
加热搅拌回流 $24 \mathrm{~h}, \mathrm{TLC}$ 检测原料点消失. 蒸出大部分 溶剂, 向残留物中加入 $30 \mathrm{~mL}$ 氯仿和 $30 \mathrm{~mL} 10 \%$ 的稀盐 酸, 搅拌 $10 \mathrm{~min}$, 分出有机层, 硫酸镁干燥, 过滤, 蒸出
大部分氯仿, 加入 $15 \mathrm{~mL}$ 甲醇, 有白色沉淀生成. 过滤 出沉淀，用氯仿/甲醇重结晶，得到白色粉末化合物 $\mathbf{3}$, 产率为 $85 \%, \mathrm{TLC}$ 检测一个点. ${ }^{1} \mathrm{H}$ NMR $\left(\mathrm{CDCl}_{3}, 500\right.$ $\mathrm{MHz}) \delta: 3.53$ (d, $\left.J=13.5 \mathrm{~Hz}, 4 \mathrm{H}, \mathrm{ArCH}_{2} \mathrm{Ar}\right), 3.70$ (d, $J=$ $\left.6.0 \mathrm{~Hz}, 4 \mathrm{H}, \mathrm{NCH}_{2}\right), 4.15$ (d, $\left.J=13.5 \mathrm{~Hz}, 4 \mathrm{H}, \mathrm{ArCH}_{2} \mathrm{Ar}\right)$, $4.58\left(\mathrm{~s}, 4 \mathrm{H}, \mathrm{OCH}_{2}\right), 6.73(\mathrm{t}, J=7.0 \mathrm{~Hz}, 2 \mathrm{H}, \mathrm{ArH}), 6.87(\mathrm{t}$, $J=7.0 \mathrm{~Hz}, 2 \mathrm{H}, \mathrm{ArH}), 7.02$ (d, $J=7.0 \mathrm{~Hz}, 4 \mathrm{H}, \mathrm{ArH}), 7.10$ (d, $J=7.0 \mathrm{~Hz}, 4 \mathrm{H}, \mathrm{ArH}), 8.34$ (s, 2H, OH), 8.51 (br, 2H, $\mathrm{NH})$; MS $m / z(\%): 587.8\left(\mathrm{M}+\mathrm{Na}^{+}, 100\right)$. Anal. calcd for $\mathrm{C}_{34} \mathrm{H}_{32} \mathrm{~N}_{2} \mathrm{O}_{6}$ : C 72.32, H 5.71, N 4.96; found $\mathrm{C} 72.39, \mathrm{H}$ $5.78, \mathrm{~N} 4.87$.

\subsection{2 双杯 [4] 冠醚 4 的合成}

方法一(分步法): 由化合物 3 合成. 在氮气保护下, 向 $100 \mathrm{~mL}$ 三口烧瓶中加入 $0.282 \mathrm{~g}(0.5 \mathrm{mmol})$ 化合物 3 , $20 \mathrm{~mL}$ 干燥二氧六环, $0.275 \mathrm{~g}(0.6 \mathrm{mmol})$ 三甘醇双对甲 苯磺酸酯, $0.12 \mathrm{~g}(5 \mathrm{mmol})$ 氢化钠. 加热搅拌回流 $24 \mathrm{~h}$, TLC 检测原料点消失. 蒸出大部分溶剂, 向残留物中加 入几滴甲醇中和过量的氢化钠. 然后加入 $20 \mathrm{~mL}$ 蒸馏水 和 $20 \mathrm{~mL}$ 氯仿, 充分搅拌后静置分层, 分出有机层. 有 机层用无水硫酸镁干燥, 过滤, 蒸出大部分溶剂, 残余 物柱层析分离[硅胶 100 200 目，淋洗剂 $V$ (二氯乙烷)： $V($ 丙酮 $)=2: 1]$, 得到白色粉末为化合物 4 , 产率为 $39 \%$, TLC 检测一个点. m.p. $248 \sim 251{ }^{\circ} \mathrm{C} ;{ }^{1} \mathrm{H}$ NMR
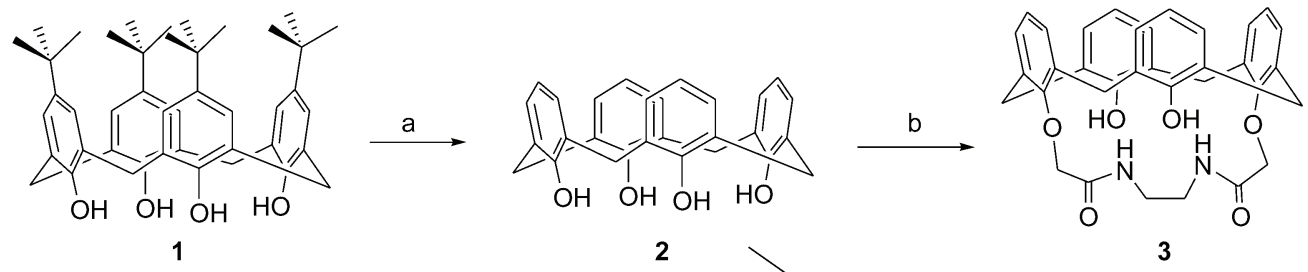

3
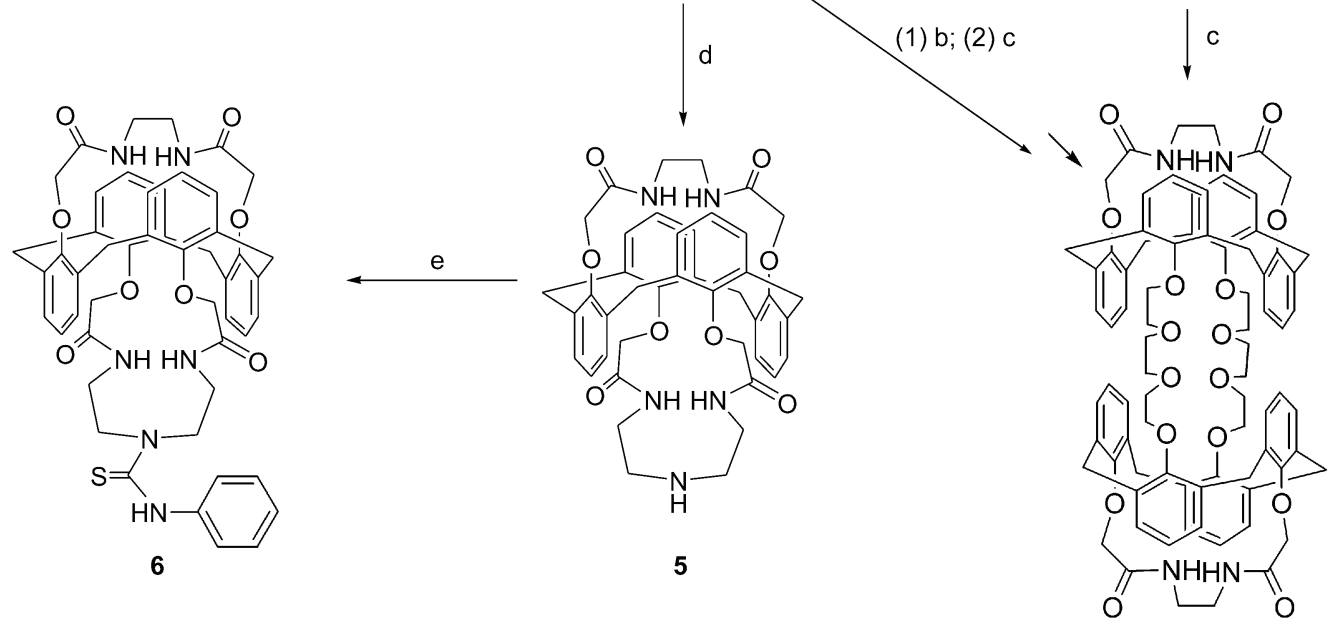

4

Reagents and conditions: (a) $\mathrm{AlCl}_{3}$, Toluene; (b) $\mathrm{ClCH}_{2} \mathrm{CONHCH}_{2} \mathrm{CH}_{2} \mathrm{NHCOCH}_{2} \mathrm{Cl}, \mathrm{K}_{2} \mathrm{CO}_{3}, \mathrm{KI}$; (c) $\mathrm{TsOCH}_{2}\left(\mathrm{CH}_{2} \mathrm{OCH}_{2}\right)_{2} \mathrm{CH}_{2} \mathrm{OTs}, \mathrm{K}_{2} \mathrm{CO}_{3}$; (d) (1) $\mathrm{ClCH}_{2} \mathrm{CONHCH}_{2} \mathrm{CH}_{2} \mathrm{NHCOCH}_{2} \mathrm{Cl}, \mathrm{K}_{2} \mathrm{CO}_{3} ;$ (2) $\mathrm{H}_{2} \mathrm{NC}_{2} \mathrm{H}_{4} \mathrm{NHC}_{2} \mathrm{H}_{4} \mathrm{NH}_{2}$; (e) PhNCS 
$\left(\mathrm{CDCl}_{3}, 500 \mathrm{MHz}\right) \delta: 3.15 \sim 4.42\left(\mathrm{~m}, 56 \mathrm{H}, \mathrm{ArCH}_{2} \mathrm{Ar}\right.$, $\mathrm{NCH}_{2}$ and $\mathrm{OCH}_{2}$ ), 6.67 $7.19(\mathrm{~m}, 24 \mathrm{H}, \mathrm{ArH}), 8.32$ (brs, $4 \mathrm{H}, \mathrm{CONH}) ; \mathrm{MS} m / z(\%): 1379.7\left(\mathrm{M}+\mathrm{Na}^{+}, 100\right)$. Anal. calcd for $\mathrm{C}_{80} \mathrm{H}_{84} \mathrm{~N}_{4} \mathrm{O}_{16}$ : C 70.78, H 6.24, N 4.13; found $\mathrm{C}$ 70.72, H 6.29, N 4.07.

方法二 (一锅法): 由化合物 2 为原料一锅法合成. 氮气保护下, 向 $100 \mathrm{~mL}$ 三口烧瓶中加入 $0.424 \mathrm{~g} \mathrm{(1}$ $\mathrm{mmol}$ )杯芳烃 2, $0.22 \mathrm{~g}(1 \mathrm{mmol}) N, N^{\prime}$-亚乙基-二(2-氯乙 酰胺), $30 \mathrm{~mL}$ 干燥丙酮, $0.55 \mathrm{~g}$ (4 mmol)无水碳酸钾, $0.50 \mathrm{~g}(3 \mathrm{mmol})$ 碘化钾. 加热搅拌回流 $24 \mathrm{~h}, \mathrm{TLC}$ 检测原 料点消失. 蒸出大部分溶剂, 然后加入 $20 \mathrm{~mL}$ 干燥二氧 六环, $0.18 \mathrm{~g}(7.5 \mathrm{mmol})$ 氢化钠, $0.46 \mathrm{~g}(1 \mathrm{mmol})$ 的三甘醇 双对甲苯磺酸酯, 搅拌回流 $24 \mathrm{~h}, \mathrm{TLC}$ 检测原料点消失. 蒸出大部分溶剂, 向残留物中加入几滴甲醇中和过量的 氢化钠. 然后加入 $20 \mathrm{~mL}$ 蒸馏水和 $20 \mathrm{~mL}$ 氯仿, 充分搅 拌后静置分层, 分出有机层. 有机层无水硫酸镁干燥, 过滤, 蒸出大部分溶剂, 残余物柱层析分离[硅胶 100 200 目, 淋洗剂 $V$ (二氯乙烷 $): V$ (丙酮 $)=2: 1$, 得到白 色粉末为化合物 4, 产率为 33\%, TLC 检测一个点. 表征 结果与方法一的产物一致.

\subsection{3 杯[4]氮杂双冠醚 $\mathbf{5}$ 的合成}

氮气保护下, 向 $100 \mathrm{~mL}$ 三口烧瓶中加入 $0.424 \mathrm{~g} \mathrm{(1}$ $\mathrm{mmol}$ )杯芳烃 2, $0.22 \mathrm{~g}$ (1 mmol) $N, N^{\prime}$-乙撑基-二(2-氯乙 酰胺), $30 \mathrm{~mL}$ 干燥丙酮, $0.55 \mathrm{~g}(4 \mathrm{mmol})$ 无水碳酸钾, $0.50 \mathrm{~g}(3 \mathrm{mmol})$ 磑化钾. 加热摚拌回流 $24 \mathrm{~h}$, TLC 检测原 料点消失. 蒸出大部分溶剂, 然后加入 $20 \mathrm{~mL}$ 二氧六环, $0.20 \mathrm{~g}(8.3 \mathrm{mmol})$ 氢化钠, $0.50 \mathrm{~g}(3 \mathrm{mmol})$ 溴乙酸乙酯. 加热摚拌回流 $24 \mathrm{~h}$. 减压蒸去大部分溶剂, 加入含 0.20 $\mathrm{g}(2 \mathrm{mmol})$ 二乙烯三胺的甲醇/氯仿溶液 $(V: V=1: 1)$ $20 \mathrm{~mL}$, 摚拌回流 $24 \mathrm{~h}$. 然后加入 $20 \mathrm{~mL}$ 蒸馏水和 $20 \mathrm{~mL}$ 氯仿, 搅拌后静置分层, 分出有机层. 有机层用 $10 \mathrm{~mL}$ $\times 3$ 蒸馏水洗涤, 无水硫酸镁干燥, 过滤, 蒸出大部分 溶剂, 残余物柱层析分离[硅胶 $100 \sim 200$ 目, 淋洗剂 $V($ 二氯乙烷 $): V($ 丙酮 $)=1: 1]$, 得到白色粉末为杯芳烃 衍生物 5, 产率 37\%, TLC 检测一个点. m.p. 264 267 ${ }^{\circ} \mathrm{C}$; ${ }^{1} \mathrm{H} \mathrm{NMR}\left(\mathrm{CDCl}_{3}, 500 \mathrm{MHz}\right) \delta: 3.10 \sim 3.27\left(\mathrm{~m}, 8 \mathrm{H}, \mathrm{NCH}_{2}\right)$, 3.82 (bs, $4 \mathrm{H}, \mathrm{NCH}_{2}$ ), 3.94 (d, $J=12.5 \mathrm{~Hz}, 4 \mathrm{H}, \mathrm{ArCH}_{2} \mathrm{Ar}$ ), 4.11 (d, $\left.J=12.5 \mathrm{~Hz}, 4 \mathrm{H}, \mathrm{ArCH}_{2} \mathrm{Ar}\right), 4.17$ (s, 4H, OCH${ }_{2} \mathrm{CO}$ ), 4.19 (s, $4 \mathrm{H}, \mathrm{OCH}_{2} \mathrm{CO}$ ), 5.23 (s, 4H, NHCO), 6.93 (t, $J=8.0$ $\mathrm{Hz}, 4 \mathrm{H}, \mathrm{ArH}$ ), 7.09 (d, $J=8.0 \mathrm{~Hz}, 8 \mathrm{H}, \mathrm{ArH}), 9.61$ (brs, $1 \mathrm{H}$, $\mathrm{NH})$; MS $m / z$ (\%): $748.7\left(\mathrm{M}^{+}, 100\right)$. Anal. calcd for $\mathrm{C}_{42} \mathrm{H}_{45} \mathrm{~N}_{5} \mathrm{O}_{8}$ : C 67.46, H 6.07, N 9.36; found C 67.38, H $6.01, \mathrm{~N} 9.25$.

\subsection{3 含硫腺基的杯 [4]氮杂双冠醚 6 的合成}

向 $100 \mathrm{~mL}$ 三口烧瓶中加入 $0.37 \mathrm{~g}(0.5 \mathrm{mmol})$ 化合物
$5,15 \mathrm{~mL}$ 氯仿, $0.1 \mathrm{~g}(0.74 \mathrm{mmol})$ 异硫氰酸苯酯. 加热搅 拌回流 $24 \mathrm{~h}$. TLC 检测化合物 $\mathbf{5}$ 消失. 蒸出溶剂, 加入 10 $\mathrm{mL}$ 石油醚, 冰箱中静置过夜, 得到白色沉淀. 过滤, 干 燥, 得到白色粉末为化合物 $\mathbf{6}$, 产率 $82 \%$, TLC 检测一个 点. m.p. $>300{ }^{\circ} \mathrm{C} ;{ }^{1} \mathrm{H}$ NMR $\left(\mathrm{CDCl}_{3}, 500 \mathrm{MHz}\right) \delta: 3.15$ (brs, $8 \mathrm{H}, \mathrm{NCH}_{2}$ ), 3.92 (brs, $8 \mathrm{H}, \mathrm{ArCH}_{2} \mathrm{Ar}$ ), 3.96 (brs, $4 \mathrm{H}$, $\mathrm{NCH}_{2}$ ), 4.16 (s, $4 \mathrm{H}, \mathrm{OCH}_{2} \mathrm{CO}$ ), 4.20 (brs, $4 \mathrm{H}, \mathrm{OCH}_{2} \mathrm{CO}$ ), 5.20 (brs, 4H, NHCO), 6.93 (t, $J=8.0 \mathrm{~Hz}, 4 \mathrm{H}, \mathrm{ArH}), 7.06$ (brs, 4H, ArH), 7.13 (d, J=9.0 Hz, 4H, ArH), 7.20 (brs, 1H, ArH), 7.35 (t, $J=8.0 \mathrm{~Hz}, 2 \mathrm{H}, \operatorname{ArH}), 7.61$ (d, $J=8.0$ $\mathrm{Hz}, 2 \mathrm{H}, \mathrm{ArH}), 8.23$ (s, $1 \mathrm{H}, \mathrm{NH})$; MS $m / z(\%): 882.3\left(\mathrm{M}^{+}\right.$, 100). Anal. calcd for $\mathrm{C}_{49} \mathrm{H}_{50} \mathrm{~N}_{6} \mathrm{O}_{8} \mathrm{~S}: \mathrm{C} 66.65, \mathrm{H} 5.71, \mathrm{~N}$ 9.51; found C 66.59, H 5.77, N 9.42.

\section{2 结果与讨论}

在合成双杯 [4]冠醚 4 的过程中, 我们最初设计方案 采用分步法先合成化合物 $\mathbf{3}$, 然后再合成化合物 $\mathbf{4}$, 以中 等产率得到产物. 由于合成化合物 $\mathbf{3}$ 时副产物少, 产率 很高, 于是我们尝试通过 “一锅法” 直接由化合物 2 合 成化合物 4 . 反应过程中不分离出中间化合物 $\mathbf{3}$, 而是采 用分步先后加入桥联试剂 $N, N^{\prime}$-乙撑基-二(2-氯乙酰胺) 和三甘醇双对甲苯磺酸酯的方法, 亦可以中等产率得到 化合物 4. 有意思的是, 不管是分步法还是一锅法, 都 只分离到 “ $2+2$ ” 的分子间缩合产物 4 , 没有检测到分 子内 “ $1+1$ ” 缩合的杯 [4]双冠醚产物. 受 “一锅法” 合 成化合物 $\mathbf{4}$ 的启发, 我们用 “一锅法”分步先后加入 $N, N^{\prime}-$ 乙撑基-二(2-氯乙酰胺)、溴乙酸乙酯和二乙烯三胺等试 剂顺利合成了含不同桥联链的杯 [4]双冠醚5, 产率中等. 反应中溴乙酸乙酯和二乙烯三胺的量都要适当过量, 否 则反应难以完全. 化合物 $\mathbf{5}$ 进一步与异硫氧酸苯酯加成, 高产率得到带硫脲基支链的杯 [4]双冠醚 6, 这种带支链 的杯 [4]双冠醚文献少见报道. 与文献合成含不同桥联 链的杯 [4]双冠醚必须采用的分步合成法相比, 本文的 分步添加桥联试剂的 “一锅法” 步骤较为简单, 产物也 易于分离, 产率中等, 是值得借鉴的一种新的合成含不 同桥联链的杯 [4]双冠醚的新方法.

所有目标化合物均经元素分析、质谱、核磁共振谱 图等表征证明为 Scheme 1 所示的结构与构象. ESI-MS 谱图中出现相应的分子离子基峰，说明预期的缩合反应 彻底完成. 核磁氢谱中, 化合物 3 的 $\mathrm{Ar}-\mathrm{CH}_{2}-\mathrm{Ar}$ 为 $1: 1$ 的一对双峰, 说明其采取 1,3-桥联模式且为杯式构象, 与文献的报道也是一致的 ${ }^{[5]}$. 由于质谱已确证了新化合 物 4,5 和 6 的桥联模式, 根据文献结构类似的化合物的 报道 ${ }^{[15 ~ 18]}$, 它们只能采取 1,3 -交替式得到杯 [4]双冠醚和 双杯 [4]冠醚. 这种去叔丁基杯 [4]芳烃在桥联反应中发生 
构象翻转得到 1,3-交替桥联产物的报道在文献中已被广 泛报道 ${ }^{[11 ~ 18]}$. 核磁氢谱中, 虽然化合物 4 由于 $\mathrm{ArCH}_{2} \mathrm{Ar}$ 与桥联链上多种 $\mathrm{CH}_{2}$ 的质子峰交叠较难分辨, 但化合物 $\mathbf{5}$ 和 6 的 $\mathrm{ArCH}_{2} \mathrm{Ar}$ 清楚表现为单峰或化学位移相近的一对 双峰，与文献报道的 1,3-交替式构象的谱图特征是一致 的 ${ }^{[1518]}$. 这些新型含不同桥联链的杯 [4]双冠醚和双杯 [4] 冠醚的性能研究正在进行当中.

\section{References}

[1] Liu, Y.; You, C. C.; Zhang, H. Y. Supramolecular Chemistry, Nankai University Publication, Tianjin, 2001, pp. 351 356 (in Chinese).

(刘育, 尤长城, 张衡益, 超分子化学, 天津南开大学出版社, 2001, pp. 351 356.)

[2] Asfari, Z.; Böhmer, V.; Harrowfield, J.; Vicens, J. Calixarenes, Kluwer Academic Publishers, Netherlands, 2001, pp. 130 154.

[3] Bondy, C. R.; Loeb, S. J. Coord. Chem. Rev. 2003, 240, 77.

[4] Chen, Y. Y.; Yang, F. F.; Lu, X. R. Tetrahedron Lett. 2000, 41, 1571.

[5] Zheng, Q. Y.; Chen, C. F.; Huang, Z. T. Chin. J. Chem. 2000, 18, 104.

[6] Casnati, A.; Ca, N. D.; Sansone, F.; Ugozzoli, F.; Ungaro, R. Tet- rahedron 2004, 60, 7869 .

[7] Oueslati, I.; Thuéry, P.; Shkurenko, O.; Suwinska, K.; Harrowfield, J.; Abidi, R.; Vicens, J. Tetrahedron 2007, 63, 62.

[8] Tang, F. S.; Yang, F. F.; Huang, Z. S.; Hong, B. Q.; Guo, H. Y. Chin. J. Org. Chem. 2009, 29(8), 1278 (in Chinese).

(唐富胜, 杨发福, 黄智胜, 洪碧琼, 郭红玉, 林建荣, 有机化学, 2009, 29(8), 1278.)

[9] Yang, F. F.; Zheng, X. H.; Guo, H. Y.; Liu, C. H.; Guo, Y. J. Inclusion Phenom. Macrocyclic Chem. 2008, 62, 371.

[10] Oueslati, I. Tetrahedron, 2007, 63, 10840.

[11] Guan, B.; Gong, S. L.; Wu, X. J.; Li, Z. H.; Chen, Y. Y. J. Inclusion Phenom. Macrocyclic Chem. 2006, 54, 81.

[12] Chawla, H. M.; Singh, S. P.; Upreti, S. Tetrahedron 2007, 63, 5636.

[13] Asfari, Z.; Nicoille, X.; Vicens, J. J. Inclusion Phenom. Macrocyclic Chem. 1999, 33, 251.

[14] Asfari, Z.; Thuery, P.; Nierlich, M.; Vicens, J. Tetrahedron Lett. 1999, 40, 499.

[15] Chawla, H. M.; Singh, S. P.; Upreti, S. Tetrahedron 2006, 62, 9758.

[16] Bitter, I.; Grün, A.; Balazs, B.; Töth, G.; Horvath, G.; Töke, L. Synth. Commun. 1999, 29, 3905.

[17] Bok, J. H.; Kim, H. J.; Lee, J. W.; Kim, S. K.; Choi, J. K.; Vicens, J.; Kim, J. S. Tetrahedron Lett. 2006, 47, 1237.

[18] Kim, H. J.; Kim, S. H.; Kim, J. H.; Anh, L. N.; Lee, J. H.; Lee, C. H.; Kim, J. S. Tetrahedron Lett. 2009, 50, 2782.

(Lu, Y.; Zheng, G.) 